\title{
Variable Refrigerant Flow (VRF) Systems in the South-Eastern United States
}

\author{
Omkar Gadgil and Scott W. Kramer, Ph.D. \\ Auburn University \\ Auburn, Alabama
}

\begin{abstract}
This research study endeavored to evaluate the use of VRF systems in the South-Eastern United States. More specifically, to understand and analyze why these systems have not been popular in this part of the world. The VRF technology, invented by the Japanese, has been around for more than 30 years. Europe and Asia-Pacific regions are two biggest consumers of this technology with their combined market share of $65 \%$ of the global market. The research aimed to get answers to three basic questions (1) What is the condition of commercial VRF market in the South-Eastern United States? (2) Why have VRF Systems not been popular in the U.S.? and (3) What is the future path for this technology in the U.S.? A survey questionnaire was carefully developed to understand and analyze -What is the extent of penetration of VRF technology; What is the level of awareness about this technology; and what types of projects are being preferred for VRF systems. The results of this survey were evaluated along with other literature including reports from analysts and interviews of leading VRF manufacturers.
\end{abstract}

Keywords: VRF Systems, HVAC, Energy Efficient Air Conditioning Systems, Refrigerant

\section{Introduction}

Air-Conditioning systems have been a part of the built environment since pre-historic times. The basic concept behind air conditioning is said to have been applied in ancient Egypt, where reeds were hung in windows and were moistened with trickling water. The evaporation of water cooled the air blowing through the window. Use of modern air-conditioning started in the $19^{\text {th }}$ century and since then, the technology has evolved to a great extent. In 1902, the first modern electrical air conditioning unit was invented by Willis Carrier in Buffalo, New York. (Wikipedia)

Air conditioning and refrigeration are provided through the removal of heat. Heat can be removed through radiation, convection, or conduction. Refrigeration conduction media such as water, air, ice, and chemicals are referred to as refrigerants. Similarly, in colder climates, heating can be achieved by transfer of heat by convection, conduction, or radiation. Before the invention of modern HVAC systems, two most popular systems used for HVAC were (and in the U.S., still are) - Direct Expansion or DX systems for residential uses and Chilled Water based Systems for commercial and industrial uses. Traditionally, heating systems were kept separate from the cooling systems and were based on use of hot water, steam or heating coil in a loop throughout the buildings or houses. However, with the help of technological advancements, we now use systems that provide heating or cooling from the same system, based on the zonal requirements. These systems have been more popular and today, we see most of the residential and commercial market using DX or Chilled Water based systems with air circulated through ducts.

Chilled Water based systems with ducted air-flow have been the most common HVAC systems used in commercial projects throughout the world. The technology for these systems was first developed in the United States and later adopted by the 
rest of the world. However, with invention of modern HVAC systems such as VRF, most of Asia-Pacific and Europe started realizing their advantages. According to a report by Pacific Northwest National Laboratory (Thornton \& Wagner, Dec 2012) VRF had about $24 \%$ of the global commercial air conditioning market, and over $35 \%$ market share in China, India, the European Union, and Eastern Europe. In 2015, the VRF market in APAC and Europe combined had a share of close to $65 \%$ (more than $\$ 7$ billion in total $\$ 11$ billion) of global market (Markets\&Markets, 2016). Hence, we can say that, today, VRF system is the most popular HVAC system for commercial use in developing parts of the world like China and India as well as developed parts like Europe and Japan. United States, on the other hand, in year 2012 (GSA, 2012) had VRF systems serving only 3\% of its office spaces which marginally increased to 4\% in 2013 and remained same for 2014. Their share in the global VRF market in year 2015 was just over $11 \%$ at $\$ 1.27$ billion (Markets\&Markets, 2016). The U.S. HVAC market, obviously, did not adopt this technology and has still been using the traditional systems as the preferred technology for its HVAC needs.

\section{Research Objective}

Commercial buildings account for approximately $40 \%$ of the energy bills and $40 \%$ of the carbon dioxide emissions in the United States (USDOE 2012a). Even today, these figures are in the range of 37\% to 40\%. About a third of commercial building energy usage is for heating, cooling and ventilation (GSA 2012). Retail sales of electricity in last 4 years (2013 to 2016) state that Commercial sector has consumed about 1.35 to 1.36 million KWH every year (US Energy Information Administration). Based on this data, we can understand how important it is for the U.S. to implement energy efficient HVAC systems, such as VRF, in this sector. The objective of this study is to understand VRF Systems market in the U.S., find out the reasons behind its slow growth and explore the possibilities of this technology being popular in next few years with focus on commercial buildings sector in the South-Eastern United States.

\section{Background}

Before we discuss further about use of VRF system in the United States, let us first understand what VRF systems are and what are their advantages and limitations over the conventional systems.

\section{History of VRF:}

VRF Systems have been in the global market for last 30 years. Daikin Industries Ltd. (Japan) is considered as one of the important players in the development of VRF systems market. The company invented variable refrigerant volume (VRV ${ }^{\mathrm{TM}}$ ) systems in 1982 in Japan. However, the real advancement in this technology was made in 1990 when modularity and simultaneous heating and cooling were made possible (Daikin). It was only in the mid-2000s, however, that these systems were ready to perform at extreme hot and cold climates as well as control humidity. During this period of 15 years, other Japanese manufacturers such as Toshiba, Mitsubishi, Panasonic etc. developed this technology and started marketing their products globally.

In her article for the ES Magazine in February 2015, Marcia Karr of WSU mentioned that, this variable refrigerant capacity technology was first conceived in the United States. However, the patent was allowed to expire when they were not successful in achieving their goal. Japanese manufacturers bought the patent and perfected the concept and introduced the ductless mini split system to the HVAC industry. (Karr, 2015)

What is the Technology?

VRF HVAC systems include two major parts, a compressor unit and multiple indoor fan coil units. The compressor unit, typically located on the roof, cools and heats refrigerant connected through piping to condition the building. The compressor units are typically air cooled. Sometimes water-cooled units are used and are connected to a cooling tower and boiler. In most installations in the United States, these systems are capable of simultaneously cooling some zones, and heating others. These systems can recover heat from spaces being cooled for use in spaces being heated and vice versa. The compressor unit uses variable refrigerant flow and is controlled by a variable-speed drive, which may operate more efficiently than conventional compressors of similar size. The complexity of the variable refrigerant flow compressor and controls results in significantly more expensive compressor units than comparable conventional systems. 
Required outside air must be delivered to the space through another mechanism. This is usually done with a separate HVAC unit, commonly called a dedicated outside air system (DOAS). These units often include energy recovery from the exhaust air to the incoming outside air, including pre-cooling the outside air when it is hot and pre-heating the outside air when it is cold, and may also recover energy used to dehumidify or humidify the outside air. (Thornton \& Wagner, 2012)

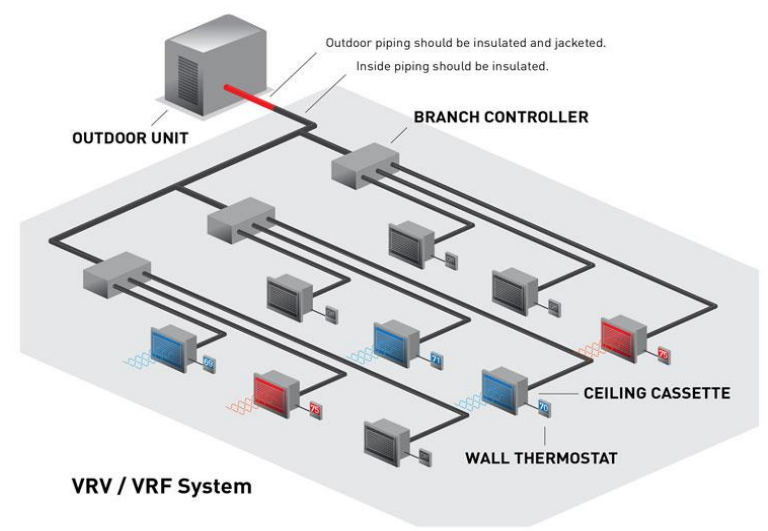

Figure 1: Schematic drawing of VRF system showing single outdoor unit with multiple indoor units. (ACHR News)

\section{System Configuration:}

Indoor units range in size from less than 1 ton up to approximately 8 tons. These units can be a fan coil type; either ducted or non-ducted, an exposed ceiling mounted unit, a concealed ceiling mounted unit or a wall mounted unit. Outdoor units range in size from approximately 5 tons for a single unit, up to 30 tons for multiple units connected together to form one single larger tonnage unit. Example: (2) 5-ton units can be connected to form a single 10-ton unit or an 8-ton unit can be connected to a 10-ton unit to yield a total tonnage of 18 tons and an 8-ton unit can be combined with a 10-ton unit and another 10-ton unit to yield a total tonnage of 28 tons. While being configured from multiple units and requiring separate power connections, the resulting "combined" unit is considered a single unit. (R\&W Engineering)

\section{Refrigerants:}

Even though the invention of refrigerant happened in early 1900s, these refrigerants were mostly toxic and flammable. The first non-flammable, non-toxic chlorofluorocarbon gas, (Freon ${ }^{\mathrm{TM}}$ ) was created by Thomas Midgley, Jr., in 1928. The blend most used in direct-expansion home and building comfort cooling is an HCFC known as chlorodifluoromethane (R-22). However, it was only in the 1990s, ozone depleting properties of these CFC gases were discovered. In mid 1990s, Germany, amongst other EU nations, was the first to ban this refrigerant followed by the U.K. in year 2000 (Wikipedia). But the U.S. continued using it for several years. Only in 2011 did the E.P.A. (EPA - Montreal Protocol) finally decide in favor of the ozone and climate-safe refrigerant for U.S. manufacture. Modern refrigerants such as R-717, R-744 $\left(\mathrm{CO}_{2}\right)$, and R-32 etc. have been developed to be more environmentally safe.

\section{$\underline{\text { Advantages of VRF system: }}$}

It is important to understand how VRF technology can be used for higher energy savings. However, this system has other advantages which, when compared with conventional systems, make VRF a better option for almost all types of applications. Below are some prominent features of VRF system...

\section{Energy Efficiency:}

One of the first research publications on VRF Technology by William Goetzler (Goetzler, 2007) for ASHRAE Journal mentions that VRF Systems use about 30\% to $40 \%$ less energy than comparable chilled water systems. Higher energy efficiency of these systems has, since then, been a topic of interest for many researchers. In last 10 years (2007-2017) several independent analyses have been carried out and almost all of them, including GSA, have confirmed this data. 
In 2013, a comparative analysis of VRF systems as alternative for conventional HVAC systems was presented (Jaesuk Park, Georgia Tech, 2013) This study investigated two aspects of VRF systems: efficient energy performance and economic benefits. VRF systems reduce energy consumption for heating and cooling by an average of $39.9 \%$ compared to conventional HVAC systems.

\section{Installation Advantages:}

VRF system components are modular, small and lightweight compared to conventional HVAC system components. They are typically installed without the use of a crane, saving substantially on installation cost. The unit modularity supports building a system over time to serve floors as a building is occupied. The low weight can reduce the need for structural reinforcement to support more massive equipment. The compressor units are typically installed outdoors and do not need a machine room or mechanical penthouse (Goetzler, 2007 as cited in Thornton \& Wagner, GSA, 2012).

\section{Space Saving:}

VRF systems have limited space requirements, particularly for the distribution system inside the building. Transferring heat through refrigerant piping requires a lot less space than ductwork. This makes VRF systems well suited to retrofits, particularly historic buildings that may not have any ductwork or cooling. In new buildings, the low space requirement can result in reduced floor-to-floor height, providing initial cost savings (Thornton \& Wagner, GSA, 2012)

Jared Edwards, CEO of HES, Dallas stated in his article for CES Magazine in August 2014, that "one of the most valuable features of VRF systems is their ability to be low profile and ductless". Moreover, most manufacturers can compact 4 tons of cooling in one single cassette unit which can be placed in a $2 \times 2$ lay-in grid. This allows us to obtain the cooling capacity of a data or equipment room by using space of only a few ceiling tiles. To reduce the outdoor footprint, most manufacturers can provide a network of up to 64 indoor units connected to one outdoor unit.

\section{Comfort:}

The most prominent advantage of VRF systems is their ability to provide heating and cooling simultaneously. This can be achieved by using the heat recovery type VRF system. The buildings can now recover wasted heat by capturing the heat normally discharged to the atmosphere in cooling mode and moving that energy to the zones that need heat.

\section{Control:}

VRF systems incorporate sophisticated controls and automation; control of the complex refrigerant system requires this. A control system is set up to communicate among all components and can operate as an energy management system within the network of systems. The control system can network with the rest of the building with open protocols such as BACnet and LONworks. Providing a comparable level of control with conventional equipment would add additional cost to those systems. As an option, the refrigerant flow to each fan coil unit can be automatically measured, allowing the energy usage of the system to be allocated to each tenant. (Thornton \& Wagner, GSA, 2012)

6. Maintenance:

Similar to conventional systems, regular maintenance of VRF systems consists of changing filters and cleaning coils for the fan coil units. Maintenance of the compressor unit is minimal, and there will be significant maintenance savings for this part of the system compared to chilled water and hot water plant equipment. This has great potential in lowering the Life Cycle Costs of the systems.

\section{Other significant features:}

In addition to the advantages mentioned above, flexibility and modularity, Lower installation time, quiet operation, LEED certification are other significant features due to which a building owner gets convinced. .

\section{Limitations of VRF system:}

\section{Refrigerant:}

Quantity - In the U.S., ANSI/ASHRAE Standard 15 limits the quantity of refrigerant per thousand cubic feet of interior volume beyond which acute toxicity is expected. Those designing VRF/VRV systems should verify the amount of refrigerant that could be lost due a leak and the smallest space into which this refrigerant could accumulate are in compliance with this standard.

Leaks - A leak in a VRF system is a serious and potentially dangerous matter. VRF/VRV systems contain much more refrigerant compared to hydronic systems. Large refrigerant leaks can require immediate evacuation of the building and possible intervention of Hazmat teams. (Seigenthaler, 2016) All leading manufacturers of VRF systems now offer advanced leak detection technology. 


\section{Fresh Air:}

Every VRF System needs to have the ability to handle fresh-air and ventilation loads as prescribed by ASHRAE 62.1/62.2. This can be achieved by introducing a separate system called DOAS (Dedicated Outside Air System) as mentioned above. This usually requires ducts to circulate the fresh air.

\section{Proprietary Systems:}

Components are not compatible across manufacturers, and there is no secondary market for components. Building owners are a captive customer once the system is installed, exposing them to a lack of price competition for replacement parts and future building retrofit projects. Manufacturers are often involved in the design of VRF systems. Structuring bid documents for open bidding of VRF systems is difficult because of manufacturer involvement in the final design. (Thornton \& Wagner, GSA, 2012)

\section{Higher equipment costs:}

VRF Systems have traditionally been considered as more expensive than chilled water systems. Jeff Ledsinger of Bartos Industries in Houston, Texas shared his thoughts about this notion in an interview with ACHR News in November 2014 issue: "When VRF is compared to other technologies on an equipment basis, just equipment versus equipment, it's usually going to come in higher because there are more components and they are a little bit more advanced electronically," Ledsinger continued. "But, then you start factoring in labor savings, the savings on controls, and other savings. All of that adds up to a lower upfront cost than most competitive technologies.

\section{The United States Market}

VRF Systems entered the U.S. market in early 2000s beginning with Mitsubishi Electric in 2003 followed by others. Daikin introduced VRV units in 2005 with products imported from Japan and Europe (Today's AC and Refrigeration news, 2017). Even though, the pioneers of air-conditioning, Carrier formed a joint venture with Toshiba in 1999, they introduced the VRF systems in North America only in the year 2012. (Carrier VRF 2016). Fujitsu General started offering their VRF products in North America in 2011. Another significant event in the U.S. VRF market would be players like Johnson Controls and Hitachi joining hands in October 2015. Johnson Controls has huge market share in the U.S. which, when coupled with Hitachi's state-of-the-art VRF technology, can be a game changer.

\section{Key Players in the U.S. VRF Market:}

The VRF Systems market in the U.S. have traditionally been dominated by Japanese manufacturers. However, in last 5 to 7 years, some new manufacturers have set up their shops here. The list below provides a snap-shot of VRF system manufacturers / suppliers in the U.S.

- Toshiba-Carrier VRF Systems (United Technologies)

- Fujitsu General

- Trane by Ingersoll-Rand

- Panasonic Corporation

- Mitsubishi Electric

- Daikin North America

- Johnson Controls - Hitachi VRF

- $\quad$ LG Electronics

- Samsung

- Lennox Commercial

Most of the above-mentioned manufacturers / suppliers have their manufacturing facilities in China, Japan and other parts of South-East Asia. However, Daikin, after acquiring Goodman, has recently started manufacturing their VRV ${ }^{\mathrm{TM}}$ equipment in a large facility in Houston, Texas.

\section{Research Methodology}

Further to our understanding of the VRF systems along with their advantages and the U.S. market conditions, this research required more detailed analysis of factors contributing or affecting the growth of this technology in this region. During last 5 years (2011 to 2016), some independent analysts have tried to carry out surveys and investigations and have published their findings. Literature about these findings was obtained and reviewed as a part of this research. As a part of this literature, 
extracts from several engineering news articles focusing on VRF technology, in which, several key people from leading VRF manufacturers were interviewed, were also reviewed (Appendix-1). One of the goals of this research was to consolidate this information, analyze and present it in a more logical manner with focus on a specific region. However, findings from these reviews needed to be re-substantiated, especially when the focus is on a specific region. One of the best ways of collecting first-hand information was to reach out to the leading engineers and contractors who design and install HVAC systems in this region. This was achieved by conducting a survey in which engineers and contractors active in the South-Eastern United States provided their responses (Appendix-2). The questionnaire of this survey was carefully drafted to understand and analyze the following...

- What is the extent of penetration of VRF technology?

- What is the level of awareness about this technology?

- What types of projects are being preferred for VRF systems?

\section{Data Analysis}

The questionnaire was targeted towards HVAC industry professionals, more specifically the designers/ engineers and contractors. Some of the responses received were from the leading A/E firms practicing in the South-East. Few responses from the U.S. Army Corps of Engineers (USACE) were quite interesting and their policies regarding the use of VRF System were understood. As the questionnaire was prepared in 2 parts, the analysis presented below is in 2 sections.

\section{Geography and Volume of Projects:}

- Respondents to the survey have been practicing in almost all South-Eastern states but more notably in Florida, Georgia and Tennessee.

- Almost all respondents' organizations are practicing for more than 25 years in the HVAC industry.

- Number of commercial projects (combined) completed by the respondents in last 5 years is $\mathbf{6 7 0 0 .}$

- Out of these, only on $\mathbf{1 0 9}$ projects the VRF Systems were installed. This corresponds to only $\mathbf{1 . 6 \%}$ of the total volume.

- Average tonnage value for these projects was found to be about 50 tons.

\section{VRF Systems' Market \\ Share in the South-East}

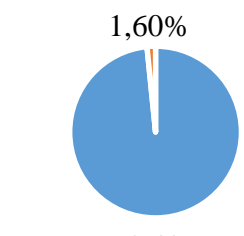

$98,40 \%$

- Conventional Systems

- VRF/VRV Systems
VRF Systems' Market
Share in the U.S.

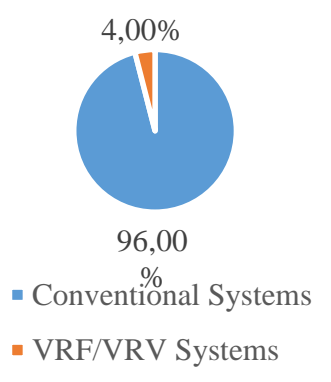

\section{Level of awareness about VRF Technology:}

- Respondents stated two main reasons due to which a VRF system can be proposed on a project...

1. Energy efficiency $-30 \%$ to $40 \%$ lower energy costs.

2. Space saving advantages - Ductless and machine room less system

- On the other hand, many different reasons were given by the respondents for not choosing this system over a traditional chilled water based systems. More notably the following...

1. Complexity of the system

2. Unavailability of trained installers and service technicians

3. Compliance with ASHRAE standards for fresh air and refrigerant concentration 
- Most of the respondents are familiar with only 1 or 2 manufacturers which provide a solution for extreme cold weather conditions.

- Out of various categories of projects, the most popular choices to propose a VRF systems were...

1. Light commercial projects

2. Refurbishment or retro-fit projects

3. Multi-family residential projects

- $\quad$ For commercial projects, multi-tenanted small office projects were chosen as more suitable for VRF systems.

- $\quad$ Out of 10-11 leading VRF brands, Toshiba, Mitsubishi, and Daikin were found to be the most popular choices.

Responses from the U.S. Army Corps of Engineers specifically pointed towards the current policies they have adopted. According to the directives received in March 2017, the Air-Force has stopped permitting VRF systems on their projects. The Army, though allowing the use, has strongly discouraged VRF Systems. On the other hand, the Navy has posed no restrictions but has asked for compliance with ASHRAE 15 safety standards for refrigeration systems. In addition to this, the USACE has also pointed out that using proprietary systems is not permitted. Most VRF manufacturers have closed proprietary control systems. The Unified Facilities Criteria (UFC) requires an open protocol system. It states that "An Open DDC system is characterized by the ability for any qualified entity to readily modify, operate, upgrade, and perform retrofits on the DDC system." (USACE, Mar 17)

\section{Conclusion}

The results of this research study have been quite convincing. Let us conclude this research by answering 3 main questions of this research paper.

1. What is the condition of commercial VRF market in the South-Eastern United States?

2. Why have the VRF Systems not been popular in the U.S.?

3. What is the future path for this technology in the U.S.?

At the outset, let us agree that the results of this research clearly state that VRF Systems have not been popular in this part of the world. As mentioned earlier, the market share of VRF systems in the U.S., as a whole, have only been $4 \%$. In the South-East, this was found to be even lower at merely $1.6 \%$.

After analyzing the responses on section-B of the survey questionnaire, we can conclude that there's sufficient awareness about the advantages of VRF technology among leading HVAC engineers. However, they are not preferring this system due to issues related to availability of trained technicians, complexity of the system, in addition to compliance to standards. Moreover, the USACE, other than compliance, is also concerned about the VRF systems being proprietary. This means, having different control protocols for different buildings for the same client is a challenge that VRF manufacturers need to tackle.

Respondents' choice of projects on which VRF could be the ideal solution were light commercial (multi-tenant offices), multi-family residential and retro-fit projects. The first two choices state that modularity and flexibility of design are important factors. Retro-fit projects, obviously, are chosen to get VRF Systems due to space-saving and duct-less solutions they offer.

Several factors need to be considered to conclude on why the VRF systems have not been popular in the South-East. My conclusion below, is not limited only to the analysis of data received from the survey. Careful consideration has also been given to what VRF industry leaders have to say about the American HVAC market as well as other literature reviewed, as mentioned above. Hence, following can be concluded as reasons for a very limited growth of this technology in the SouthEast. 


\section{Geography and Climate:}

The U.S. has been geographically divided in various regions viz. North, North-East, West, Mid-West, South-East etc. Out of these, the North, North-East and the West regions have seen higher commercial growth. Out of these three regions, the North-East has seen rapid growth during the second industrial revolution. Development in the West and the South is comparatively recent. We can, hence, infer that while majority of South-East was being developed, the conventional chilled water systems were the best solution. The structures were planned for this and till date most of them function quite well. This, along with North-East predominantly falling under colder climatic regions, have been very important factors to consider. VRF systems have, traditionally, not been considered suitable for colder climates.

Another important geographical factor to consider is that the U.S. is located quite far away from the manufacturing facilities of leading VRF providers. Except for a very recent development by Daikin to manufacture VRF machines in their Texas plant, all other providers manufacture their machines in Asia. This causes longer delivery times and most commercial projects have very limited construction timelines. On the other hand, most chillers and AHUs are manufactured locally.

\section{Timing:}

As stated above, VRF systems entered the U.S. market in the new millennium. By the time this technology started picking up some market share, the recession took over. This, arguably, has impacted VRF's growth, to a great extent, more specifically due to higher initial costs. Even after 2010, the technology didn't quite get the luxury of asking for a premium Cap-Ex, especially in a more conservative economic growth. Since 2010, VRF has seen considerable amount of growth in the West and the North-East. However, the South-East, with an exception of cities like Atlanta, GA, Nashville, TN and Raleigh-Durham, NC, has not been growing so rapidly. Moreover, by the time VRF systems entered the market, most of the commercial developments were planned for conventional ducted air-flow systems. These buildings have not yet reached a stage for refurbishment.

\section{Economics:}

One of the major factors to limit the growth of VRF in the South-East is economics. This can be concluded with two main points, Energy-Economics and Space-Economics. Energy prices have historically been lower than the national average in this region. Due to this, energy efficiency aspect of VRF systems has not been quite convincing for new projects. Easy availability of water for cooling needs would also be a factor worth mentioning. In addition to the energy factor, space availability of commercial projects in the South-East has been much more than other crowded cities in the North-East and the West. Hence, the space saving advantage of VRF systems has not yet been a decision maker. This, of course, is changing in the bigger cities like Atlanta. The economic conditions in the West and North-East are quite the opposite. Energy rates are higher, space is at a premium and hence, VRF systems are seeing better prospects in these regions.

\section{Standards and Regulations:}

Standards and regulations for HVAC industry are primarily governed by ASHRAE, AHRI and U.S. Department of Energy. Out of several standards and regulations, the ones that have impacted the growth of VRF's market share are...

- ASHRAE Standard 15 which regulates refrigerant safety and environmental impacts and

- ASHRAE Standard 62 for Ventilation and Indoor Air Quality which states the requirement of outside air (fresh air)

Both, the survey respondents as well as USACE have raised concerns about VRF systems' compliance to these standards. This clearly indicates that the VRF industry needs to work on solutions to overcome this issue. On the bright side, VRF systems have shown excellent results against ASHRAE's standards for minimum efficiency ratios including full-load energy-efficiency ratio (EER) values, and part load, integrated energy-efficiency ratio (IEER) values. (Thornton \& Wagner, GSA, 2012)

Finally, we need to figure out what the future holds for this technology in the U.S. Industry leaders in VRF market are very optimistic about its future. Independent market research reports also point towards a bright future for VRF systems in the U.S. According to the analysts at Markets\&Markets, the VRF systems market in North America was valued at US\$ 1.27 billion in 2015 and it is expected to reach US\$ 3.16 Billion by 2022 at a CAGR of 13.5\%. This, of course, is dependent on the commercial construction activity which is increasing since last few years. (Markets\&Markets, 2016).

As the utility prices keep increasing, more and more requirements for lowering operating costs will call for choosing VRF systems over conventional chilled water systems. This has been evident in growth of VRF sales in North-East and western 
states like California, Arizona, New Mexico etc. where utility prices are above normal. Another important factor for growth in VRF systems sale in the North-East is that many office and government buildings are now 60-80 years old and don't have duct-work. In the South-East, however, VRF manufacturers need to work on various challenges stated above, in order to increase VRF's market share.

To summarize, VRF systems have not been popular in the South-Eastern United States. Main challenges the VRF manufacturers need to address are including but not limited to the following...

- Increase trained installers and technicians base

- Spread awareness about energy savings

- Provide better solutions for ASHRAE compliance

- Decrease equipment lead times

- Develop and offer open protocol solutions

\section{References}

Wikipedia on Air-Conditioning Retrieved from https://en.wikipedia.org/wiki/Air_conditioning

Variable Refrigerant Flow Systems by Brian Thornton and Anne Wagner, report prepared for the General Services Administration (GSA) by Pacific Northwest National Laboratory in December 2012. Retrieved from https://www.gsa.gov/portal/mediaId/197399/fileName/GPG_Variable_Refrigerant_Flow_12-2012.action

Variable Refrigerant Flow GPG-006, December 2012 Published by General Services Administration through their GPG (Green Proving Ground) program on their website. Retrieved from https://www.gsa.gov/portal/content/163491

Global report on VRF Systems by Markets and Markets July 2016. Retrieved from http://www.marketsandmarkets.com/Market-Reports/variable-refrigerant-flow-systems-market-155809495.html

U.S. Energy Information Administration website provides interactive data browser https://www.eia.gov/electricity/data/browser/

Daikin - History of VRV Innovation https://www.daikin.com/about/why_daikin/vrv/index.html

Variable Refrigerant Flow is Evolving by Marcia L. Karr, P.E. for Engineered Systems Magazine February 2015 issue. Retrieved from http://www.esmagazine.com/articles/96976-vrf-is-evolving? $\mathrm{v}=$ preview

Don Pfaff, P.E. and Kristin Trone, R\&W Engineering Blog Post on Variable Refrigerant Flow (VRF) Systems, October 2015. Retrieved from http://rweng.com/blogpost/vrf-systems/

Comparative Analysis of The VRF System and Conventional HVAC Systems, Focused On Life-Cycle Cost by Jaesuk Park, Georgia Institute of Technology, 2013. Retrieved from https://smartech.gatech.edu/bitstream/handle/1853/50227/PARK-THESIS-2013.pdf

EPA (Environmental Protection Agency 2010) https://www.epa.gov/ods-phaseout/phaseout-class-ii-ozone-depletingsubstances

Water Trumps Refrigerant by John Siegenthaler, P.E. for Supply House Times, December 2016 Pg. 32-34

01-VRF-011-01.pdf. (n.d.). Retrieved from http://www.utcces-cdn.com/hvac/docs/1001/Public/0C/01-VRF-011-01.pdf

Today's AC and Refrigeration news, Feb 2017 Retrieved from http://todays-ac.com/wpcontent/uploads/2017/02/EntireFebruary2017Issue.pdf

HVRF Systems. http://www.modbs.co.uk/news/archivestory.php/aid/12445/VRF_meets_chilled_water.html

US Army Core of Engineers - Engineering and Construction Bulletin March 2017 Retrieved from https://www.wbdg.org/FFC/ARMYCOE/COEECB/ecb_2017_7.pdf 